\title{
LUT
}

Lappeenranta

University of Technology

\section{GFDM-Based Cooperative Relaying Networks with Wireless Energy Harvesting}

Carrillo Melgarejo Dick, Moualeu Jules M., Nardelli Pedro, Fraidenraich Gustavo, da Costa Daniel B.

This is a Post-print

version of a publication

published by IEEE

in 2019 16th International Symposium on Wireless Communication Systems (ISWCS)

DOI: $\quad 10.1109 / I S W C S .2019 .8877135$

Copyright of the original publication: (C) Copyright 2019 IEEE

Please cite the publication as follows:

Carrillo Melgarejo D., Moualeu J. M., Nardelli P., Fraidenraich G., da Costa D. B.(2019). GFDMBased Cooperative Relaying Networks with Wireless Energy Harvesting. Published in: 2019 16th International Symposium on Wireless Communication Systems (ISWCS), Oulu, Finland, 27-30 Aug. 2019. DOI: 10.1109/ISWCS.2019.8877135 


\title{
GFDM-Based Cooperative Relaying Networks with Wireless Energy Harvesting
}

\author{
Dick Carrillo Melgarejo ${ }^{1}$, Jules M. Moualeu ${ }^{2}$, Pedro Nardelli ${ }^{1}$, Gustavo Fraidenraich ${ }^{3}$, and Daniel B. da Costa ${ }^{4}$ \\ ${ }^{1}$ School of Energy Systems, LUT University, Finland \\ ${ }^{2}$ School of Electrical and Information Engineering, University of the Witwatersrand, South Africa \\ ${ }^{3}$ School of Electrical and Computer Engineering, University of Campinas, Brazil \\ ${ }^{4}$ Department of Computer Engineering, Federal University of Ceará, Sobral, Brazil \\ e-mail: \{dick.carrillo.melgarejo,pedro.juliano.nardelli\}@lut.fi, jules.moualeu@wits.ac.za, \\ gf@decom.fee.unicamp.br, danielbcosta@ieee.org
}

\begin{abstract}
In this paper, the concept of wireless power transfer and wireless information transmission, which have recently received special attention for improving energy efficiency in wireless communication systems, are investigated. In particular, we focus on a cooperative communication network consisting of a source node, an energy-constrained relay node and a destination node. In contrast to conventional wireless-powered cooperative networks, a GFDM waveform-which is an emerging candidate waveform for the 5G mobile networks and beyond-is considered. The performance of the proposed GFDM-based relaying network with energy harvesting is studied in terms of the average BER for the general $\mathcal{M}$-QAM constellation set. Numerical and simulation results are provided to give useful insights and to assess the accuracy of our mathematical derivations.
\end{abstract}

Index Terms-Energy harvesting, GFDM ,relay networks, BER, SWIPT.

\section{INTRODUCTION}

In recent years, many wireless technologies have adopted the orthogonal frequency division multiplexing (OFDM) scheme as a popular scheme for encoding digital data over multiple carrier frequencies. The use of such a waveform dates back from the introduction of the standard IEEE 802.11 (Wi-Fi) until the 3rd Generation Partnership Project (3GPP) release 16, which is an evolution of the actual Long-Term Evolution (LTE) technology that aims to support the fifth generation $(5 \mathrm{G})$ of cellular connectivity [1], [2]. This preference is based on the fact that OFDM makes efficient use of the spectrum, is computationally efficient to implement modulation and demodulation functions with the adoption of fast Fourier transform (FFT) techniques, and is relatively simple from a receiver implementation viewpoint over various adaptive equalization techniques [3]. However, OFDM has some disadvantages that have been well reported in the current literature. For instance, high peak-to-average power ratio (PAPR), sensitivity in intercarrier interference (ICI), and the inefficiency of the cyclic prefix (CP) that fails when the delay distribution in the channel is longer than the $\mathrm{CP}$ length, are just some limitations of the aforementioned waveform [4]. In an effort to address these limitations, various techniques have been proposed to support new wireless technologies such as $5 \mathrm{G}$ and beyond. One, that has particularly received a lot of interest recently, is the generalized frequency division multiplexing (GFDM). GFDM is a non-orthogonal waveform that can transmit multi-symbols per multi-carrier in a twodimensional block structure i.e., time and frequency, and which can be achieved by a circular convolution of each sub-carrier through pulse shaping [5], [6]. To overcome fading channels, it dynamically adopts pulse shaping optimization in time and frequency. All the aforementioned features enable GFDM to achieve spectrum and energy efficiency when compared to OFDM. However, GFDM also presents some disadvantages. For example, in some cases, the effects of ICI and intersymbol interference (ISI) are significant since the variable pulse shaping filters remove orthogonality between the sub-carriers.

One important design objective for $5 \mathrm{G}$ and beyond wireless networks is energy efficiency. To overcome the bottleneck of energy-constrained wireless devices, a promising solution that prolongs their battery lifetime-making them self-sustainable -has recently been proposed through energy harvesting (EH). Traditional energy harvesting techniques rely on the surrounding environment using various natural sources such as wind, solar, and thermal just to name a few. However, such EH methods are often unstable due to the unpredictability of the weather conditions, and therefore, are not suitable to provide perpetual and ubiquitous energy demands. To this end, an alternate approach that relies on radio frequency (RF) signals has been proposed. RF signals, which are widely considered as information carriers, can also be used as a vehicle for transporting energy [7]-[9]. In other words, RF signals become a potential source of wireless power transfer (WPT) and wireless information transfer (WIT). In light of this, a simultaneous wireless information and power transfer (SWIPT) scheme, which is based on the simultaneous transport of energy and information through the ambient RF signals, has been regarded as a sustainable approach [10].

Due to the versatility of RF signals to carry both energy and information, a SWIPT technique is naturally applicable to dual-hop cooperative relaying networks which have been widely investigated in the current literature [10]-[12]. In that context, a relay device is kept active without relying on conventional power sources. Current studies on SWIPT relay 
architectures consider either OFDM or other general waveform models with multiple-input multiple-output (MIMO) setups. However, the above-mentioned waveforms lack some degree of flexibility necessary to address all the requirements for the 5G technology. To this end, to further support flexibility in terms of resource allocation while improving the quality of the user experience and improving the battery lifetime of an energy-constrained device, a GDFM transmission can be incorporated in an EH-based cooperative relaying network. In [13], a GFDM-based cooperative decode-and-forward (DF) relaying network is proposed. In that work, the relay node performs WIT and WPT to the destination node using different GFDM sub-block sets. However, such a setup (with GFDM and $\mathrm{EH}$ receivers at the destination) has some limitations due to the high implementation cost associated with computational resources. Thus, this renders work of [13] less attractive for energy-constrained devices such as internet-of-things (IoT) devices or wireless sensors.

To the best of the authors' knowledge, one can affirm that there is no work on GFDM-based amplify-and-forward (AF) cooperative EH-relaying with SWIPT. To partly fill this gap, we propose a GFDM and EH-based cooperative AF relaying network, wherein the relay node scavenges energy from the source node in order to forward the source information to the destination node which employs a minimum mean square error (MMSE) receiver. Unlike in [13], the energy-constrained relay node employs an AF protocol, and therefore, does not require to perform any demodulation of the GFDM signal. Moreover, we derive an analytic bit error rate (BER) expression of the proposed system for a general $\mathcal{M}$-quadrature amplitude modulation (QAM) constellation set. Monte Carlo simulations are provided in order to assess the accuracy of the proposed mathematical derivations.

The remainder of this paper is structured as follows. Section II describes the system model considered in this work. In Section III, the performance of the proposed system is investigated by deriving an expression of the average BER. Numerical and Monte Carlo simulations are provided in Section IV, and, finally, Section V gives some concluding remarks.

\section{SYSTEM MODEL}

Consider a wireless network shown in Fig. 1, consisting of a base station, $S_{t}$, an energy-constrained relay node, $R$, and a mobile user, $D$. All the nodes are equipped with a single antenna. It is assumed that no direct link exists between $S_{t}$ and $D$ due to poor channel conditions or heavy shadowing. To this end, the transmission of GFDM symbols takes place in two time slots. In the first time slot, $S_{t}$ transmits to $R$, wherein EH by employing a SWIPT scheme is used over a high signal-to-noise ratio (SNR) regime. Then, $R$ amplifies the received signal from the source and forwards to $D$ in the second time slot. It is assumed that all the received signals are corrupted by additive white Gaussian noise (AWGN) and the fading channels are assumed to be quasi-static. It is worthwhile mentioning that the channel state information (CSI) is known at the receiver. A detailed description of the system model is

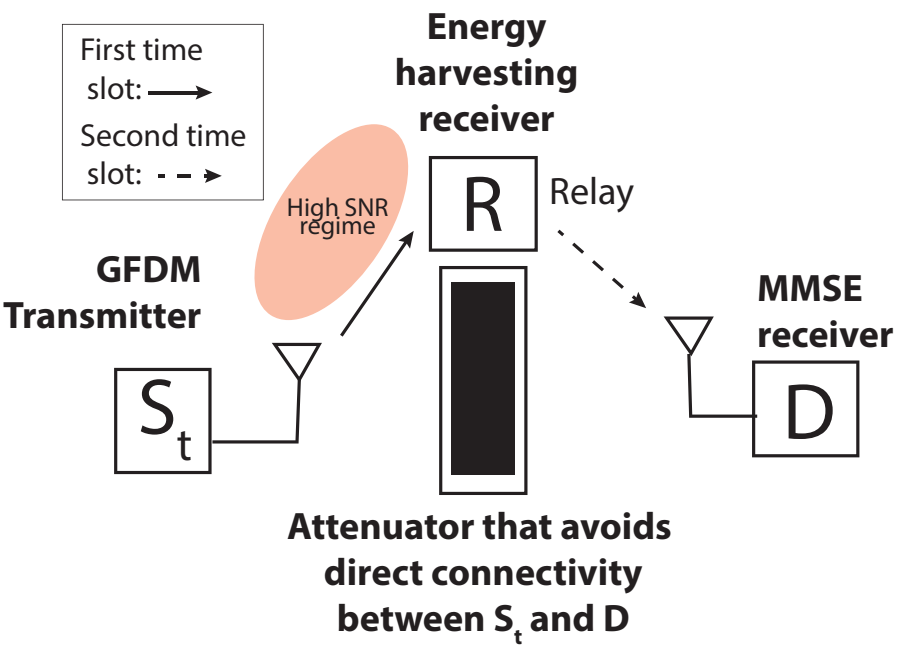

Fig. 1. Illustration of a GFDM-based cooperative EH-relay system.

presented subsequently. In what follows, we briefly revisit the concept of GFDM followed by the one on SWIPT.

\section{A. GFDM system}

The system is designed to transmit a complex symbol block $d_{s, k}$ from $S_{t}$ to $D$ at the $s$ th time instance and $k$ th subchannel containing $S \times K$ data symbols, $s=0, \ldots S-1$, $k=0, \ldots K-1$. We assume that data symbols are independent and identically distributed (i.i.d.). In this regard, the GFDM signal can be given by

$$
x[n]=\sum_{s=0}^{S-1} \sum_{k=0}^{K-1} d_{s, k} g_{s, k}[n],
$$

in which $g_{s, k}[n]$ is the circular time-frequency shifted version of the prototype filter $g[n]$ expressed as

$$
g_{s, k}[n] \triangleq g\left[(n-s K)_{N}\right] e^{j 2 \pi n k / K},
$$

where $N=S \times K$ and $(.)_{N}$ is the modulo operator. Note that, in order to facilitate a circular convolution, the transmitter filter $g[n]$ is circular with a period of $n \bmod N$. It is also important to remark that in (2), the GFDM shifting step is $K$ and $1 / K$ in time and frequency domains, respectively. Next, the transmitter and receiver blocks are described .

1) Transmitter: The modulated signal in (1) can be further expressed in a matrix notation form, wherein all the elements of the transmit symbol block are organized in a single vector as $\mathbf{d}=\left[\mathbf{d}_{0}^{T}, \ldots \mathbf{d}_{s-1}^{T}\right]^{T}$, in which $s=0, \ldots S-1$, and $\mathbf{d}_{s}=$ $\left[\mathbf{d}_{s, 0}, \ldots \mathbf{d}_{s, K-1}\right]^{T}$ with variance $\sigma_{\mathrm{d}}^{2}$. The vector form of $x[n]$ for $n=0, \ldots N-1$ can be written as

$$
\mathbf{x}=\mathbf{A d},
$$

where $\mathbf{x}=[x[0], \ldots x[N-1]]^{T}$ and $\mathbf{A}$, of dimension $N \times N$, is the modulation matrix or self-interference matrix of GFDM defined as $\mathbf{A}=\left[\mathbf{G}_{0}, \ldots \mathbf{G}_{S-1}\right]$, in which $\mathbf{G}_{s}$, 
for $s=0, \ldots, S-1$, is the $N \times K$ matrix of $g_{s, k}[n]$ coefficients, such that

$$
\mathbf{G}_{s}=\left[\begin{array}{cccc}
g_{s, 0}[0] & g_{s, 1}[0] & \cdots & g_{s, K-1}[0] \\
g_{s, 0}[1] & g_{s, 1}[1] & \cdots & g_{s, K-1}[1] \\
\vdots & \vdots & \ddots & \vdots \\
g_{s, 0}[N-1] & g_{s, 1}[N-1] & \cdots & g_{s, K-1}[N-1]
\end{array}\right] .
$$

A cyclic prefix (CP) of length $N_{\mathrm{cp}}$ is added to the GFDM signal $\mathbf{x}$ to prevent inter-block interference over a frequencyselective channel (FSC). Therefore, the transmitted signal is given by $\mathbf{x}_{\mathrm{cp}}=\left[\mathbf{x}\left(N-N_{\mathrm{cp}}+1: N\right) ; \mathbf{x}\right]$.

2) Receiver: We assume a zero-mean circular symmetric complex (ZMCSC) Gaussian Channel $\mathbf{h}=\left[h_{1}, h_{2}, \cdots, h_{L}\right]^{T}$ where $h_{r}$, with $1 \leqslant r \leqslant L$, represents the complex baseband channel coefficient of the $r$ th path. We also consider that $N_{\text {cp }} \geqslant L$, and the channel coefficients related to different paths are uncorrelated. The received vector of length $N_{\mathrm{t}}=N_{\mathrm{cp}}+N+L-1$ is given by $\mathbf{y}_{c p}=\mathbf{h} * \mathbf{x}_{\mathrm{cp}}+\nu_{\mathrm{cp}}$, where the symbol $(*)$ denotes the linear convolution, $\nu_{\mathrm{cp}}$ is the AWGN vector of length $N_{\mathrm{t}}$ with variance $\sigma_{\nu}^{2}$. On the receiver side, $\mathrm{CP}$ is removed. As a consequence, the frequencydomain equalization (FDE) properties can be exploited, and the linear convolution in (5) yields to a circular convolution. The resulting vector becomes

$$
\mathbf{y}=\mathbf{H}_{\mathrm{ch}} \boldsymbol{A} \mathbf{d}+\nu,
$$

where $\nu$ is the AWGN vector of length $N$ with variance $\sigma_{\nu}^{2}$ and $\mathbf{H}_{\mathrm{ch}}$ is the $N \times N$ circular Toeplitz matrix based on vector $\mathbf{h}$ and given as described in [14] by

$$
\mathbf{H}_{\mathrm{ch}}=\left[\begin{array}{ccccccc}
h_{1} & 0 & \cdots & 0 & h_{L} & \cdots & h_{2} \\
h_{2} & h_{1} & \cdots & 0 & 0 & \cdots & h_{3} \\
\vdots & & \ddots & & & \cdots & \vdots \\
h_{L} & h_{L-1} & \cdots & \cdots & \cdots & \cdots & 0 \\
0 & h_{L} & \cdots & \cdots & \cdots & \cdots & 0 \\
\vdots & & \ddots & & & \cdots & \vdots \\
0 & 0 & & h_{L} & \cdots & \cdots & h_{1}
\end{array}\right] .
$$

In order to estimate the transmitted complex data symbols, we considered a MMSE receiver matrix $\left(p \mathbf{I}_{N}+\right.$ $\left.\left(\mathbf{H}_{\mathrm{ch}} \mathbf{A}\right)^{\dagger}\left(\mathbf{H}_{\mathrm{ch}} \mathbf{A}\right)\right)^{-1}\left(\mathbf{H}_{\mathrm{ch}} \mathbf{A}\right)^{\dagger}$, in which the operator $(.)^{\dagger}$ represents the Hermitian-conjugate of a matrix, $\mathbf{I}_{N}$ is a $N \times N$ identity matrix, and $p$ is the average SNR given as $p=\sigma_{d}^{2} / \sigma_{\nu}^{2}$. Conditioned on the matrix $\left(\mathbf{H}_{\mathrm{ch}} \mathbf{A}\right)$, the signal-to-interferenceand-noise ratio (SINR) on the $n$th data symbol can be expressed as

$$
\gamma_{n}=\frac{1}{\mathrm{MMSE}_{n}}-1=\frac{1}{\left[\left(\mathbf{I}_{N}+\frac{p}{N}\left(\mathbf{H}_{\mathrm{ch}} \mathbf{A}\right)^{\dagger} \mathbf{H}_{\mathrm{ch}} \mathbf{A}\right)^{-1}\right]_{n n}}-1 .
$$

Note that (7), given in the same form as [15, Eq. 7.49], is derived based on the second-order statistics of the input signals, not restricted to binary signals [16]. The -1 term in (7) is to account for bias [17].

\section{B. TSR Protocol}

Here, we consider an ideal scenario where the information transfer receiver and the $\mathrm{EH}$ at the relay can operate simultaneously, and have access to the total received signal and its energy. 1

In the time-switching relaying (TSR) protocol, the node $R$ switches between information detection and $\mathrm{EH}$ during the transmission block $T$. Let $v$ denote the percentage of the block time $T$ to perform simultaneously information reception and energy harvesting on the node $R$ with $0 \leqslant v<1$. In second half of the block time $(1-v) T, R$ re-transmits the received signal from the source to $D$ using AF.

1) Source-to-relay information and power transfer: In this work, it is assumed that both information and energy receivers are designed to operate at the same location [19]. Therefore, the information transmission and energy transfer are characterized by the same channels M. Other important assumption on the system model is that the matrix $\mathbf{M}$ is composed by the product of an identity matrix ${ }^{2} \mathbf{I}$ and a constant channel gain $m$ defined by $\mathbf{M}=m \mathbf{I}$. For the case of information transfer, the received vector $\mathbf{y}$ at the relay node is given by Eq. (5) and Eq. (3),

$$
\mathbf{y}_{r}=\mathbf{M x}+\nu_{r}
$$

where $\mathbf{x}$ is defined in (3), and the noise is defined by the AWGN vector $\nu_{r}$ with zero mean and variance equal to $\sigma_{r}^{2}$.

For the case of energy transfer, the $\mathrm{EH}$ receiver converts the RF signal $\mathbf{y}_{r}$ to a direct current (DC) using a rectifier without the need for the conversion from RF band to baseband [19]. So, the harvested energy at the relay, in units of energy, is given by

$$
Q_{r}=\frac{1}{2} \zeta \mathbb{E}\left[\|\mathbf{M} \mathbf{x}\|^{2}\right]=\zeta \operatorname{tr}\left(\mathbf{M P}(\mathbf{M})^{\dagger}\right)
$$

where $\zeta \in[0,1]$ is the conversion efficiency and $\operatorname{tr}(\cdot)$ denotes the trace operator. The matrix $\mathbf{P}$ is the covariance of the vector $\mathbf{x}$ given by $\mathbb{E}\left[\mathbf{x x}^{\dagger}\right]$. As aforementioned, $d_{s, k}$ is a GFDMgenerated data symbol, and the filter prototype power in (1) is assumed to be $g_{s, k}[n]=1$. Thus, the variable $\mathbf{P}$ is an identity matrix, and as a result, the harvested energy $Q_{r}$ will depend only on the channel matrix $\mathbf{M}$. The used energy at the relay is defined as $E_{u}$ and is either varying or constant given by

$$
E_{u}= \begin{cases}\bar{E}_{u}, & \text { if the used energy is constant, } \\ \beta Q_{r}, & \text { otherwise, }\end{cases}
$$

where $\beta$ is the ratio of the harvested energy to the used energy, with $0 \leqslant \beta \leqslant 1$, and $E_{u} \leqslant Q_{r}$. According to previous definitions, the transmit power at the relay node using the whole frame transmission size is given by

$$
P_{r}=2 E_{u}
$$

${ }^{1}$ In the existing literature, many practical schemes that separate WPT and WIT have been proposed. One of these schemes is known as power splitting, which is developed on the power domain [18], while the other scheme based on the time domain is known as time switching [7].

${ }^{2}$ Justified because the source-to-relay system is operating on a high SNR regime, which is an ideal condition for $\mathrm{EH}$ 
As stated in [18], we assume an infinite capacity storage on relay node. In this work, and without loss of generality, it is assumed that the power at the energy-constrained relay node is constant i.e., $E_{u}=\bar{E}_{u}$.

2) Relay-to-destination transmission: The received vector at the destination is defined by $\mathbf{y}_{d}$ given by

$$
\mathbf{y}_{d}=\mathbf{H}_{\mathrm{ch}} \mathbf{y}_{r}+\nu_{d}
$$

After substituting (8) in (12), the following is obtained

$$
\mathbf{y}_{d}=\mathbf{H}_{c h} \mathbf{M x}+\mathbf{H}_{c h} \nu_{r}+\nu_{d}
$$

where $\nu_{d}$ is the AWGN vector whose entries are i.i.d. with zero mean and variance equal to $\sigma_{d}^{2}$. The signal $\mathbf{y}_{d}$ should be constrained by the gain transmission at the relay node, as elaborated subsequently.

\section{Power transmission gain in the relay}

Based on the previous consideration, in (12), it is assumed that the term $\mathbf{H}_{\mathrm{ch}} \nu_{r}$ is not significant compared to $\nu_{d}$ i.e., $\sigma_{r}^{2} \ll \sigma_{d}^{2}{ }^{3}$. In addition, the term $\mathbf{H}_{c h} \mathbf{M x}$, that represents the product of a circular Toeplitz matrix $\mathbf{H}_{\mathrm{ch}}$ with matrix $\mathbf{M}$ results in a circular Toeplitz matrix $\mathbf{M}_{c h}$, similar to the matrix defined in (6).

Another important fact to consider in the system model is that the EH-relay system considers the TSR protocol, which was explained before. Based on the aforementioned discussions, the received signal at the destination node $D$ can be simplified to

$$
\mathbf{y}_{d} \approx \mathbf{M}_{c h} \mathbf{x}+\nu_{d}
$$

where the power gain at the relay node is defined as

$$
g_{v}= \begin{cases}(1-v) P_{r}, & \text { scenario } 1, \\ (1-v)(v) P_{r}, & \text { scenario } 2 .\end{cases}
$$

with scenario 1 representing the case in which the energy stored in the relay battery is always guaranteed, available, and potentially the RF-EH system is complemented by other EH system as for example photo-voltaic source. In this way, the power gain used to transmit from the relay node depends only on the frame window available for transmission, which is equal to $1-v$. In scenario 2 , which is a more economical case, the power gain used for energy transfer depends on the portion of the frame used for EH and the portion of the frame used for information transmission i.e., $(v)$ and $(1-v)$. In both scenarios, the power gain is always constant, because $v$ and $P_{r}\left(E_{u}\right)$ are constant, and $E_{u} \leqslant Q_{r}$. Since it is assumed a constant power transmission in relay, increasing the period for harvesting implies in a smaller gain for $g_{v}$.

\footnotetext{
${ }^{3}$ Such an assumption is practical since the relay node is an internet of things (IoT) device operating on a high SNR regime, and therefore, its receiver noise power can be negligible in comparison to the additive noise at the destination node.
}

\section{Performance Analysis}

\section{A. BER calculation}

Assuming a $\mathcal{M}$-QAM constellation, the conditional BER denoted by $P_{b}\left(E \mid \gamma_{n}\right)$, is approximated using a general mathematical formulation [20]. The average BER is given by

$$
P_{b}(E)=\frac{1}{N} \sum_{n=0}^{N-1} \int_{0}^{\infty} P_{b}\left(E \mid \gamma_{n}\right) p_{\gamma}\left(\gamma_{n}\right) d \gamma_{n}
$$

where with the help of (14) and the constraint of (15), the probability density function (PDF) $p_{\gamma}\left(\gamma_{n}\right)$ can be approximated by [21]

$$
p_{\gamma}\left(\gamma_{n}\right) \approx \frac{1}{\Gamma(\kappa) \theta^{\kappa}}\left(1+\gamma_{n}\right)^{-1-\kappa} \exp \left(-\frac{1}{\left(1+\gamma_{n}\right) \theta}\right),
$$

where $\theta=\sigma^{2} / \mu$ and $k=\mu^{2} / \sigma^{2}$,

$$
\begin{aligned}
& \mu=\mathbb{E}\left[\alpha_{n}\right]=-\sum_{j=1}^{N}\left[\frac{1}{\Psi_{j}^{2}} \exp \left(\frac{N}{\Psi_{j}^{2}}\right) \operatorname{Ei}\left(-\frac{N}{\Psi_{j}^{2}}\right)\right], \\
& \sigma^{2}= \mathbb{E}\left[\alpha_{n}^{2}\right]-\left(\mathbb{E}\left[\alpha_{n}\right]\right)^{2} \\
&= \sum_{j=1}^{N}\left[\frac{1}{N \Psi_{j}^{2}}+\frac{1}{\Psi_{j}^{4}} \exp \left(\frac{N}{\Psi_{j}^{2}}\right) \operatorname{Ei}\left(-\frac{N}{\Psi_{j}^{2}}\right)\right. \\
&\left.-\frac{1}{\Psi_{j}^{4}} \exp \left(\frac{2 N}{\Psi_{j}^{2}}\right) \operatorname{Ei}^{2}\left(-\frac{N}{\Psi_{j}^{2}}\right)\right],
\end{aligned}
$$

$\alpha_{n}=\frac{1}{\gamma_{n}+1}, \operatorname{Ei}(x)$ is the exponential integral function, and

$$
\Psi_{j}^{2}=2 p g_{v} \Phi^{2}\left|\chi_{j}\right|^{2},
$$

with $\Phi^{2}=\sum_{r=1}^{N} \sigma_{r}^{2}$. $\left|\chi_{j}\right|$ being the eigenvalue $j$ of $\mathbf{A}$ with $j=1, \cdots N$. Plugging in (18) in (16) and after performing some mathematical manipulations, the average BER can be expressed as in (17) at the top of the next page.

From (21), it can be observed that when the gain $g_{v}$ increases, the mean and the variance defined in Eq. (19) and (20), respectively, will vary, and consequently the PDF defined in (18) will also vary. Hence, the BER defined in (16) is dependent on $g_{v}$.

Our model provides detailed explanation of the BER dependency based on the PDF of $\gamma_{n}$ for different values of $v$. Fig. 3 shows the PDF of $\gamma_{n}$ for scenario 1. Here, when $v$ increases the PDF is more concentrated in low SINR values, which explains the poor performance.

The PDF of $\gamma_{n}$ is depicted in Fig. 4. In this figure, it can be seen that the PDF that is less concentrated in low values of $\operatorname{SINR}\left(\gamma_{n}\right)$, is the one that corresponds to $v=0.5$, which provides the best BER performance.

\section{Numerical Results}

In this section, some numerical results for the BER are plotted against $v$ for a fixed $\mathrm{SNR}=20 \mathrm{~dB}$ (high power regime), by considering the GFDM parameters: $K=32$ (subcarriers), $S=3$ (subsymbols), root raised cosine (RRC) as the prototype filter, roll-off factor $=0.9, L=2$, and power 


$$
\begin{aligned}
P_{b}(E) & \approx \frac{1}{N} \sum_{n=0}^{N-1} \int_{0}^{\infty} \frac{1}{\log _{2} \sqrt{\mathcal{M}}} \sum_{l=1}^{\log _{2} \sqrt{\mathcal{M}}} \frac{1}{\sqrt{\mathcal{M}}} \sum_{t=0}^{\left(1-2^{-l}\right) \sqrt{\mathcal{M}}-1}\left\{(-1)^{\left\lfloor\frac{t 2^{l-1}}{\sqrt{\mathcal{M}}}\right\rfloor} \times\left(2^{l-1}-\left\lfloor\frac{t 2^{l-1}}{\sqrt{\mathcal{M}}}+\frac{1}{2}\right\rfloor\right) \times \operatorname{erfc}\left((2 t+1) \sqrt{\frac{3 \log _{2} \mathcal{M} \gamma_{n}}{2(\mathcal{M}-1)}}\right)\right\} \\
& \times \frac{1}{\Gamma(k) \theta^{k}}\left(1+\gamma_{n}\right)^{-1-k} \exp \left(-\frac{1}{\left(1+\gamma_{n}\right) \theta}\right) d \gamma_{n}
\end{aligned}
$$

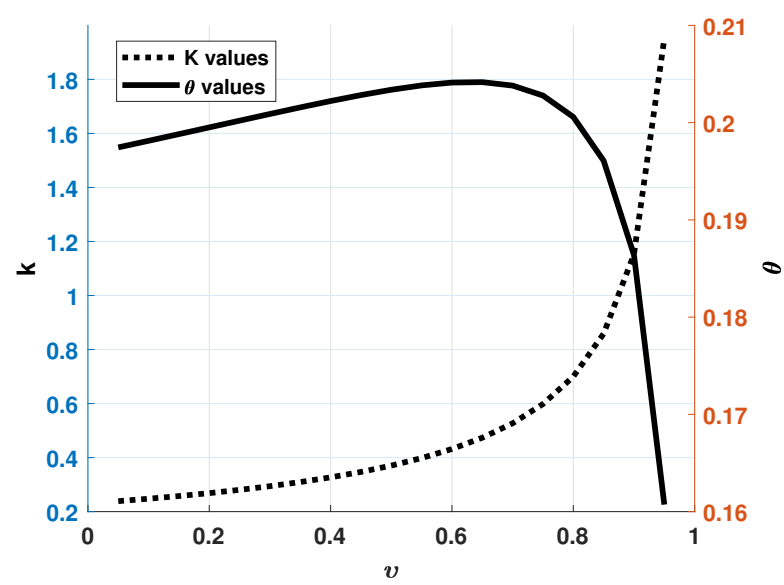

Fig. 2. Analyze of $k$ and $\theta$ for different values of $v$

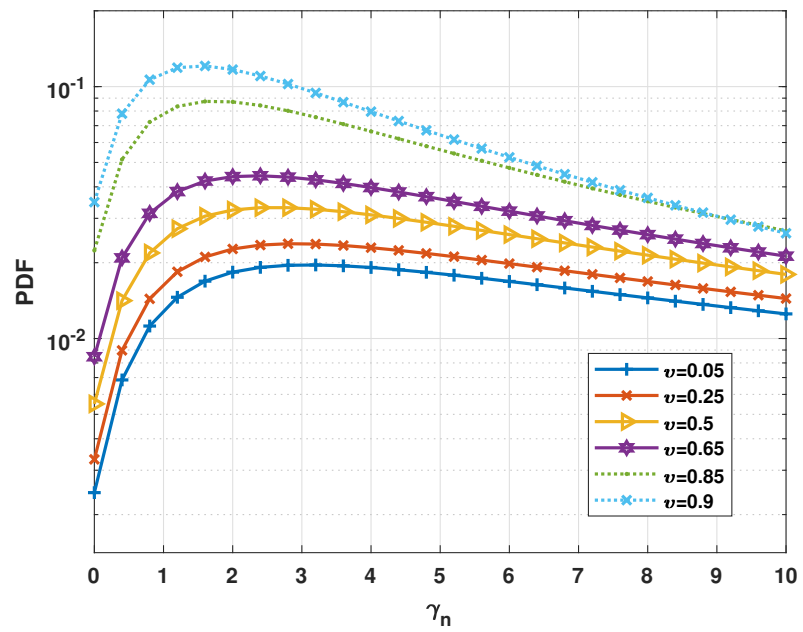

Fig. 3. PDF analysis for different values of $v$ in scenario 1 .

delay profile $\sigma_{w}^{2}=e^{-w / 5}, w=1, \ldots, N$. The channel matrix $\mathbf{M}_{c h}$ considers a frequency selective fading channel (FSFC). For the harvesting scheme, we consider a power gain of the relay node that depends directly on the frame utilization for the TSR protocol, a conversion efficiency $(\zeta=0.7)$, and a constant energy at the relay constrained by $\bar{E}_{u}<Q_{r}$.

In Fig. 5, the approximated BER versus the average SNR is presented for different values of $v$. Moreover, it is noted that the Monte Carlo simulations are in good agreement with

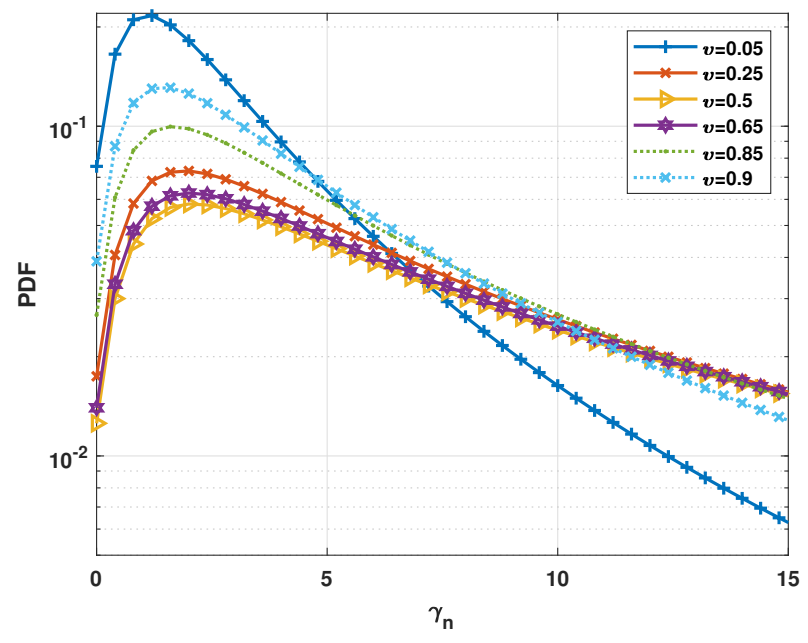

Fig. 4. PDF analysis for different values of $v$ in scenario 2 .

the proposed mathematical derivations over the entire SNR regime. The particular case of $v=0$ represents, for scenario 1 , that the relay power is independent of the RF signal. So, as much the dependency of RF harvesting, the worst condition for BER performance. Fig. 6 shows the BER performance of

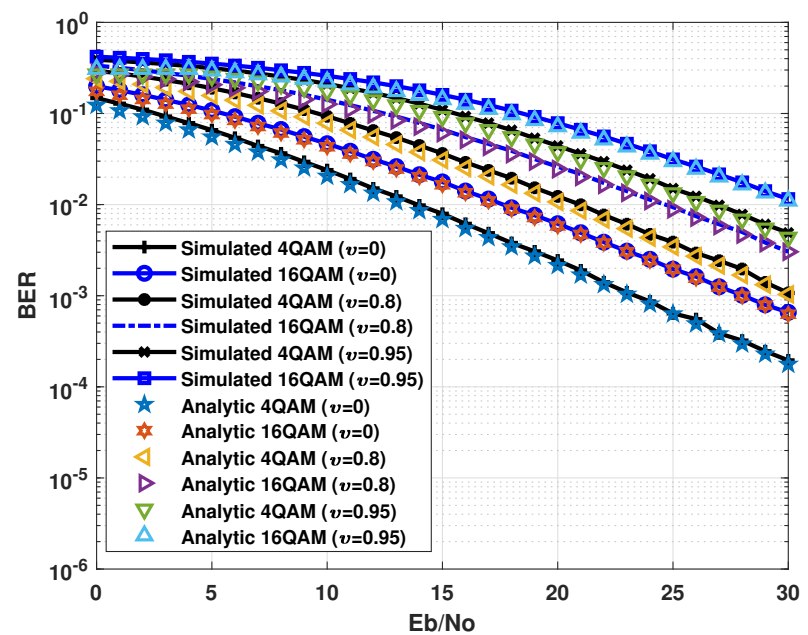

Fig. 5. BER performance versus Eb/No for GFDM-EH-relay system with three different values for $v$. 
the proposed system considering both scenarios described in (15). It can be seen that scenario 1 outperforms scenario 2 in terms of BER independent of $v$ value. However, scenario 2 represents a less expensive scenario because the power transmission only depends of the $\mathrm{EH}$ received from the $\mathrm{RF}$ signal. It is also important to mention that Monte Carlo simulations and the analytic results are in good agreement, which confirms the accuracy of the BER expression exposed in this work.

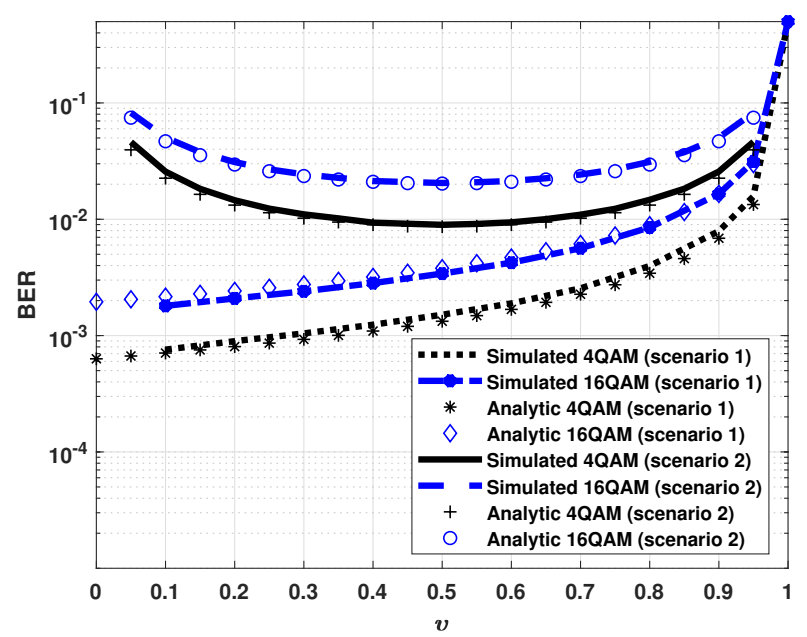

Fig. 6. BER performance versus $v$ for the GFDM-EH-relay system. Here is possible to compare scenario 1 and scenario 2 .

\section{CONCLUSiOnS}

In this paper, we considered a novel EH-GFDM-based cooperative relaying network using SWIPT scheme, TSR protocol and GFDM waveform over a FSFC with a MMSE receiver. The proposed system assumed that the available power at the relay node is always constant. Two scenarios were evaluated for the TSR scheme based on the proportional split $v$ of the frame used for SWIPT. In both cases, an analytic approximation of the BER for a general $\mathcal{M}$-QAM constellation using GFDM waveform was derived. Scenario 1, that represents a more expensive setup because it assumes any complementary EH systems to RF energy source, outperforms scenario 2. For example, for $v=0.3$ and GFDM with 4QAM the BER gain of scenario $1(B E R=0.001)$ over scenario $2(\mathrm{BER}=0.01)$ is approximately 10 . Finally, the analytic and simulation results showed a good accuracy in both scenarios.

\section{ACKNOWLEDGEMENTS}

This work is partly funded by the LUT foundation and Academy of Finland via ee-IoT project (ICT2023/n.319009) and via FIREMAN consortium (CHIST-ERA/n.326270).

\section{REFERENCES}

[1] J. Sachs, L. A. A. Andersson, J. Araújo, C. Curescu, J. Lundsjö, G. Rune, E. Steinbach, and G. Wikström, "Adaptive 5G low-latency communication for tactile internet services," Proceedings of the IEEE, vol. 107, no. 2, pp. 325-349, Feb 2019.

[2] C. L. et al., "5g-based systems design for tactile internet," Proceedings of the IEEE, vol. 107, no. 2, pp. 307-324, Feb 2019.

[3] H. Sampath, S. Talwar, J. Tellado, V. Erceg, and A. Paulraj, "A fourthgeneration mimo-ofdm broadband wireless system: design, performance, and field trial results," IEEE Communications Magazine, vol. 40, no. 9, pp. 143-149, Sep. 2002.

[4] Y. Jiang, "New companding transform for papr reduction in ofdm," IEEE Communications Letters, vol. 14, no. 4, pp. 282-284, April 2010.

[5] N. Michailow, S. Krone, M. Lentmaier, and G. Fettweis, "Bit error rate performance of generalized frequency division multiplexing," in 2012 IEEE Vehicular Technology Conference (VTC Fall), Sep. 2012, pp. 1-5.

[6] A. Farhang, N. Marchetti, and L. E. Doyle, "Low-complexity modem design for gfdm," IEEE Transactions on Signal Processing, vol. 64, no. 6, pp. 1507-1518, March 2016.

[7] R. Zhang and C. K. Ho, "Mimo broadcasting for simultaneous wireless information and power transfer," IEEE Transactions on Wireless Communications, vol. 12, no. 5, pp. 1989-2001, May 2013.

[8] A. Ammar and D. Reynolds, "Energy harvesting networks: energy versus data cooperation," IEEE Communications Letters, vol. 22, no. 10, pp. 2128-2131, Oct 2018.

[9] H. Zhang, Y. Guo, Z. Zhong, and W. Wu, "Cooperative integration of rf energy harvesting and dedicated wpt for wireless sensor networks," IEEE Microwave and Wireless Components Letters, vol. 29, no. 4, pp. 291-293, April 2019.

[10] F. Benkhelifa and M. Alouini, "Precoding design of mimo amplifyand-forward communication system with an energy harvesting relay and possibly imperfect csi," IEEE Access, vol. 5, pp. 578-594, 2017.

[11] W. Han, J. Ge, and J. Men, "Performance analysis for noma energy harvesting relaying networks with transmit antenna selection and maximalratio combining over nakagami-m fading," IET Communications, vol. 10, no. 18 , pp. 2687-2693, 2016

[12] Y. Gu and S. Aïssa, "Rf-based energy harvesting in decode-and-forward relaying systems: Ergodic and outage capacities," IEEE Trans. Wireless Commun., vol. 14, no. 11, pp. 6425-6434.

[13] Z. Na, J. Lv, M. Zhang, B. Peng, M. Xiong, and M. Guan, "Gfdm based wireless powered communication for cooperative relay system," IEEE Access, vol. 7, pp. 50971-50 979, 2019.

[14] S. Tiwari, S. S. Das, and K. K. Bandyopadhyay, "Precoded generalized frequency division multiplexing system to combat intercarrier interference: performance analysis," IET Communications, vol. 9, no. 15, pp. 1829 - 1841, May 2015.

[15] A. Paulraj, R. Nabar, and D. Gore, Introduction to space-time wireless communications. Cambridge university press, 2003.

[16] P. Li, D. Paul, R. Narasimhan, and J. Cioff, "On the distribution of SINR for the MMSE MIMO receiver and performance analysis," IEEE Trans. Inf. Theory, vol. 52, no. 1, pp. 271 - 286, Jan 2006.

[17] J. Cioffi, "EE379A: Digital communications-signal processing and EE379C: Advanced digital communications," 2018.

[18] F. Benkhelifa and M. Alouini, "Precoding design of mimo amplifyand-forward communication system with an energy harvesting relay and possibly imperfect csi," IEEE Access, vol. 5, pp. 578-594, 2017.

[19] R. Zhang and C. K. Ho, "Mimo broadcasting for simultaneous wireless information and power transfer," IEEE Transactions on Wireless Communications, vol. 12, no. 5, pp. 1989-2001, May 2013.

[20] K. Cho and D. Yoon, "On the general BER expression of one-and two-dimensional Generalized Frequency Division Multiplexing for 5th Generation Cellular Networks amplitude modulations," IEEE Trans. on Commun., vol. 50, no. 7, pp. 1074-1080, Jul 2002.

[21] D. Carrillo, S. Kumar, G. Fraidenraich, and L. L. Mendes, "Bit error probability for mmse receiver in gfdm systems," IEEE Communications Letters, vol. 22, no. 5, pp. 942-945, May 2018. 